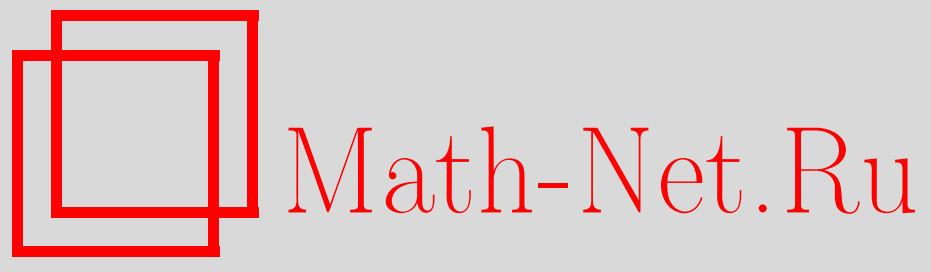

А. А. Давыдов, А. Ф. Нассар, О стационарном состоянии в динамике популяции с иерархической конкуренцией, УМН, 2014, том 69, выпуск 6, 179-180

DOI: https://doi.org/10.4213/rm9631

Использование Общероссийского математического портала Math-Net.Ru подразумевает, что вы прочитали и согласны с пользовательским соглашением http: //www. mathnet.ru/rus/agreement

Параметры загрузки:

IP: 54.196 .121 .252

26 апреля 2023 г., 12:24:41

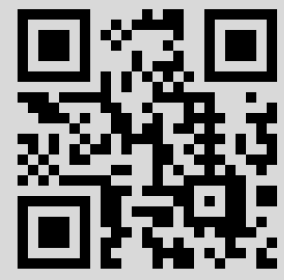




\title{
О стационарном состоянии в динамике популяции с иерархической конкуренцией
}

\author{
А. А. Давыдов, А. Ф. Нассар
}

Мы показываем, что при заданном управлении в динамике структурированной по размеру популяции с иерархической формой конкуренции есть нетривиальное стационарное состояние. Точнее, мы предполагаем, что эта динамика описывается уравнением

$$
\frac{\partial x(t, l)}{\partial t}+\frac{\partial[g(l, E(t, l)) x(t, l)]}{\partial l}=-(\mu(l, E(t, l))+u(l)) x(t, l),
$$

где $x$ - плотность индивидуумов размера $l$ в момент времени $t$, функции $g$ и $\mu$ характеризуют соответственно их рост и смертность при уровне конкуренции $E$, а управление $u$ - эксплуатацию популяции. Функция конкуренции $E$ задается формулой

$$
E(t, l)=\int_{l}^{L} \chi(s) x(t, s) d s,
$$

где $\chi$ - некоторая непрерывная функция, положительная при положительном аргументе, а отрезок $[0, L], L>0,-$ это диапазон размеров, на котором популяция эксплуатируется. В силу формулы конкуренции влияние на развитие индивидуумов данного размера имеют лишь индивидуумы не меньшего размера. Этот факт отличает данную работу от работ [1]-[3], где интеграл берется по всему отрезку $[0, L]$ и уровень конкуренции одинаков для индивидуумов всех размеров. Граничное условие имеет вид

$$
x(t, 0)=\int_{0}^{L} r(l, E(t, l)) x^{\beta}(t, l) d l+p_{0}
$$

и отвечает за приток индивидуумов размера $l=0$ : этот приток есть сумма естественного воспроизводства и промышленного возобновления $p_{0}=$ const $\geqslant 0$. Функция $r$ и показатель $\beta, \beta \in(0,1)$, характеризуют репродуктивность биомассы и нелинейную зависимость воспроизводства от плотности соответственно. Модель (1)-(3) аналогична хорошо известным (см., например, [4], [5]). Мы предполагаем, что все функции в ней, за исключением $u$, непрерывны и удовлетворяют следующим естественным условиям:

(а) при каждом $l \in[0, L]$ функции $g$ и $r$ не возрастают с увеличением конкуренции $E$, при этом функция $g$ всюду положительна, а функция $r$ всюду неотрицательна и положительна хотя бы на некотором непустом интервале из отрезка $[0, L]$;

(b) $\mu$ - положительная функция, неубывающая по $E$ при каждом $l \in[0, L]$;

(c) при $0 \leqslant l_{1}<l_{2} \leqslant L$ отношение $g\left(l_{1}, \cdot\right) / g\left(l_{2}, \cdot\right)$ не возрастает по $E$.

Условия (a)-(c) отражают неулучшение развития популяции при росте конкуренции и не меньшее влияние ее роста на индивидуумов малых размеров, чем на индивидуумов больших размеров.

Допустимым называется измеримое управление $u$, удовлетворяющее на $[0, L]$ условию $0 \leqslant u_{1} \leqslant u \leqslant u_{2}$ с некоторыми кусочно непрерывными функциями $u_{1}$ и $u_{2}$.

Теорема. Для любого допустимого управления и существует положителъное непрерывное стационарное решение задачи (1)-(3), если в слое $0 \leqslant l \leqslant L$ функиии $g, \mu, r$ удовлетворяют условиям (a)-(c), а функиии $g, \mu-$ условию Липшица no $E$.

Искомое стационарное решение должно быть решением задачи Коши

$$
\frac{d z}{d l}=-M(l, E) z, \quad \frac{d E}{d l}=-\widetilde{\chi}(l, E) z, \quad z(L)=z_{L} \geqslant 0, \quad E(L)=0,
$$

Работа выполнена при поддержке программы Президиума РАН “Динамические системы и теория управления" и программы "Ведущие научные школы" (грант НШ-5138.2014.1).

DOI: $10.4213 / \mathrm{rm} 9631$ 
где $z=g(l, E) x, M=(\mu+u) / g, \tilde{\chi}=\chi / g$, с некоторым значением $z_{L}$. В силу непрерывности функций $\mu, g$ и их липшицевости по $E$, непрерывности $\chi$, а также измеримости и ограниченности управления $u$ последняя система уравнений удовлетворяет в слое $0 \leqslant l \leqslant L$ теореме существования и единственности решения задачи Коши [6]. Решения задачи (4) являются невозрастающими функциями в силу неположительности правых частей уравнений и поэтому при продолжении влево остаются в области $z \geqslant 0$, $E \geqslant 0$. Для двух таких решений $\left(z_{1}, E_{1}\right)$ и $\left(z_{2}, E_{2}\right)$ при $0<z_{1}(L)<z_{2}(L)$ верно

$$
0<z_{1}(L) \leqslant z_{1}(l)<z_{2}(l), \quad 0 \leqslant E_{1}(l) \leqslant E_{2}(l)
$$

всюду на $[0, L]$, где эти решения оба определены, ибо для любого $l \in[0, L]$ правые части уравнений системы - неположительные невозрастающие по $z$ и $E$ функции при $z \geqslant 0$ и $E \geqslant 0$. Следовательно, при наличии продолжения такого решения до $l=0$ получим $E(0)=\phi(z(0))$ с некоторой неубывающей функцией $\phi, \phi(0)=0$, непрерывной в силу теоремы о непрерывной зависимости решения от начальных данных [6]. На таком решении отношение $d E / d z$ не превосходит максимума $m$ функции $\chi / \mu$ на отрезке $[0, L]$ при $E=0$, поскольку управление неотрицательно, а $\mu$ - неубывающая функция по $E$; поэтому $\phi(z) \leqslant m z$. Отсюда и из неравенства $z(L) \leqslant z(0)$ следует, что функция $\phi$ определена для всех $z \geqslant 0$. График функции $\phi$ в исходных переменных есть также график некоторой непрерывной неубывающей функции $\Phi, \Phi(0)=0$, поскольку $x(0)=z(0) / g(0, \phi(z(0)))$, а $g$ - невозрастающая функция $E$. Осталось показать, что существует решение с начальными данными на графике функции $\Phi$, удовлетворяющее условию (3) (здесь $\left.x_{0}=x(0)\right)$ :

$$
x_{0}=\int_{0}^{L} r(l, E(l)) x^{\beta}(l) d l+p_{0} .
$$

Правая часть равенства (5) имеет оценку снизу $c x_{0}^{\beta}$ и сверху $C x_{0}^{\beta}$ при малых и больших $x_{0}>0$ соответственно с некоторыми константами $c>0, C>0$. Следовательно, поскольку $\beta \in(0,1)$, при малых $x_{0}>0$ левая часть равенства (5) меньше правой, а при больших - наоборот. Но обе части равенства (5) непрерывно зависят от точки $\left(x_{0}, \Phi\left(x_{0}\right)\right), x(0) \geqslant 0$, поэтому существует $x_{0}>0$, когда они равны, что и означает существование стационарного решения при выбранном управлении и доказывает теорему.

Единственность такого решения - открытая проблема.

\section{Список литературы}

[1] A. A. Davydov, A.S. Platov, Mosc. Math. J., 12:2 (2012), 269-273. [2] А. А. Давыдов, А. С. Платов, Тр. ИММ УрО РАН, 19:4 (2013), 89-94. [3] А. А. Панеш, А. С. Платов, Пробл. матем. анализа, 2012, №67, 107-112. [4] L. F. Murphy, J. Theor. Biol., 104:4 (1983), 493-506. [5] A. M. de Roos, Structured populations models in marine, terrestrial and freshwater systems, eds. S. Tuljapurkar, H. Caswell, Chapman \& Hall, New York, 1997, 119-204.[6] Э.А.Коддингтон, Н. Левинсон, Теория обыкновенных дифференциалъных уравнений, ЛКИ, 2007, 476 с.

\section{А. А. Давыдов (А. А. Davydov)}

Московский государственный университет им. М. В. Ломоносова;

International Institute for Applied System Analysis,

Laxenburg, Austria

E-mail: davydov@vlsu.ru

\section{A. Ф. Haccap (A. F. Nassar)}

Владимирский государственный университет

им. А. Г. и Н. Г. Столетовых

E-mail: amer6767@mail.ru
Представлено А. Г. Сергеевым

Принято редколлегией

12.11 .2014 